\title{
Improved Monte Carlo method to Study Road Reliability
}

\author{
Fang Jian*, Sun Yaonan \\ Department of Civil Engineering, School of Science, Nanjing University of Science and Technology, Nanjing, China
}

\section{Email address:}

2664757778@qq.com (Fang Jian)

*Corresponding author

\section{To cite this article:}

Fang Jian, Sun Yaonan. Improved Monte Carlo method to Study Road Reliability. Science Discovery. Vol. 8, No. 2, 2020 , pp. $32-36$. doi: $10.11648 /$ j.sd.20200802.12

Received: February 10, 2020; Accepted: May 26, 2020; Published: June 4, 2020

\begin{abstract}
This paper firstly introduces the development of Monte-Carlo method in connectivity reliability and its advantages and disadvantages in the study of road connectivity reliability. Secondly, introduce the traditional Monte Carlo method to calculate the reliability of the connection, and improve the accuracy and speed of the traditional Monte Carlo method, that is, replace the pseudo-random number with Sobol sequence, which overcomes the The shortcomings make the result more accurate and increase the calculation speed from the side. Finally, by comparing the results of the Monte Carlo method and Monte Carlo method for calculating the reliability of road connectivity, the advantages of the improved Monte Carlo method in this respect are obtained. It shows that this method has certain correctness and superiority, and has a positive reference value for the evaluation of the reliability of the road connectivity. The research on the reliability of road connectivity, this paper improves the accuracy and speed of the traditional Monte Carlo method, that is, replacing the pseudo-random numbers with Sobol sequences, which overcomes the shortcomings caused by pseudo-random sequence sampling and makes the results It is more accurate and speeds up calculations from the side. Finally, by comparing the results of improved Monte Carlo method and Monte Carlo method for the seismic reliability calculation of roads, the advantages of improved Monte Carlo method in this respect are obtained. It shows that the method has certain correctness and superiority, and has a positive reference value for road reliability assessment.
\end{abstract}

Keywords: Connectivity Reliability, Improved Monte Carlo Method, Sobol Sequence, Accuracy

\section{改进蒙特卡洛法研究道路的连通可靠性}

\section{方剑"，孙耀南}

南京理工大学理学院土木工程系, 南京, 中国

邮箱

2664757778@qq.com（方剑）

摘要：本文在对道路的连通可靠性研究中，首先介绍了蒙特卡罗法在连通可靠性的发展以及其优缺点。其次，介绍传 统蒙特卡洛法计算连通可靠性的方法, 并对传统的蒙特卡洛法进行精确度以及速度方面的改进, 即用Sobol序列代替伪 随机数, 克服了由于伪随机序列抽样带来的缺点, 使得结果更加精确, 从侧面也提高了计算速度。最后通过改进蒙特 卡洛法与蒙特卡洛法对于道路的连通可靠性计算的结果对比, 得出改进蒙特卡洛法在此方面的优势。表明该方法具有 一定的正确性和优越性, 对道路的连通可靠性评估具有积极的参考价值。

关键词: 连通可靠性, 改进蒙特卡洛法, Sobol序列, 精确性 


\section{1. 引言}

众所周知, 生命线对于抗震救灾和重建家园非常重 要, 而其中道路抗震可靠性又是重中之重, 连通可靠性是 生命线网络如道路网络分析的关键环节, 较好的处理道路 的连通可靠性对道路抗震可靠性计算具有重要意义。随 着电子计算机以及计算方法的进步, 人们对于道路抗震可 靠性的评估越来越精确。道路抗震可靠性考虑因素众多, 蒙特卡洛法作为一种能够轻松处理复杂情况的方法而应 用广泛。文献[1]采用蒙特卡洛法, 先对城市交通路网的单 元进行可靠性分析, 然后纵观全局进行分析, 从而得到网 络连通概率。文献[2]对于节点进行了不同烈度下的分析, 采用蒙特卡络法算出了网络节点的可靠度。文献[3]系统阐 述了基于蒙特卡络方法的网络连通可靠性, 为道路抗震可 靠性研究提供了一个基本理论依据。文献[4]在采用蒙特卡 洛模拟法对山区路网连通可靠度进行分析和计算中, 通过 概率解析法来检验蒙特卡罗模拟法的计算精度, 并在文献 [2]的基础上加入了边坡和隧道的因素。文献[5]在震后通 行可靠度出发, 用蒙特卡洛法得到了最优化疏散路径。文 献[6]在文献[4]增加了震后各种道路上的各种因素, 如道 路与沿街建筑的距离。文献[7]提出了解决路网问题的一种 可靠方法, 首次提出路段概率重要度。文献 [8]应用蒙特卡 洛法时, 加上了道路单元、桥梁单元、边坡单元、隧道单 元的通行概率。文献[9]在采用蒙特卡络法分析路网抗震可 靠性时, 增加了单体的易损性分析。但是随着研究的深入, 蒙特卡洛法的弊端也暴露出来, 文献[10]提出了蒙特卡洛 模拟法也存在收玫速度慢、误差具有概率性等不足的地 方。针对传统蒙特卡洛法的弊端, 本文从精确度和速度两 方面进行优化, 提出一种改进的蒙特卡洛法, 即改进蒙特 卡洛法。在研究道路连通可靠性上, 此法较以往的蒙特卡 洛法提高了精确度和速度。

\section{2. 蒙特卡洛法与改进蒙特卡洛法}

\section{1. 蒙特卡洛法}

随着电子计算机的发展, 工程上一些必需的数值求解 问题, 包括复杂积分函数的数值积分方法、有限差分近似 离散等问题可以通过求近似解的方法来解决。蒙特卡洛法 就是求近似解的一类随机方法的统称, 而且是目前求解复 杂概率问题唯一可行途径。

蒙特卡洛法是指基于统计学中的贝努力大数定律来 得到一定容量的样本数据, 用概率模型来把数学或物理问 题相联系, 采用电子计算机来实现统计模拟或抽样, 当抽 样的次数足够多时, 则频率就等于所求解问题的概率。

在求解道路的连通可靠性时, 由于其复杂的交通网络, 概率解析法难以解决, 而蒙特卡洛法又适用于网络庞大和 结构复杂的情况, 因此本文采用蒙特卡洛法对其进行研究。

蒙特卡洛法在道路连通可靠性的应用步骤如下:

(1)首先根据道路的网络结构图, 给各个节点编号, 并 建立它们间关联矩阵 $\mathrm{R}[\mathrm{i}][\mathrm{j}]$, 用 0 到 1 之间的数来表示两个
相关节点间的可靠性数, 其中自身节点（i=j）连通可靠性 的数值设为 1 , 不相关则为 0 ;

(2)然后用计算机生成随机数矩阵: $X=[\mathrm{i}][\mathrm{j}]$;

(3)接着建立布尔矩阵并计算:

$B[i][j]=R[i][j]-X[i][j]$;

如果 $B[i][j]>0$, 则 $B[i][j]=1$;

如果 $B[i][j] \leq 0$, 则 $B[i][j]=0$;

(4)道路的网络存在二元传递关系, 可用Warshall算法 [11]的传递闭包(设 $W$ 是一个二元关系, 若 $W$ '是包含 $W$ 的最 小的传递关系, 则称 $\mathrm{W}$ '为 $\mathrm{W}$ 的传递闭包)进行计算, 可以 得到道路网络的可达矩阵BL[i][j];

(5)若 $B L[i][j]=1$, 则表示节点 $i$ 与节点 $j$ 能保持连通; 若 $B L[i][j]=0$ ，则表示节点 $i$ 与节点 $j$ 不连通;

最后将步骤(2) (5)重复 $\mathrm{N}$ 次, 其中若有 $n$ 次连通, 则节 点间的连通可靠度为:

$$
\mathrm{Q} \approx \mathrm{n} / N
$$

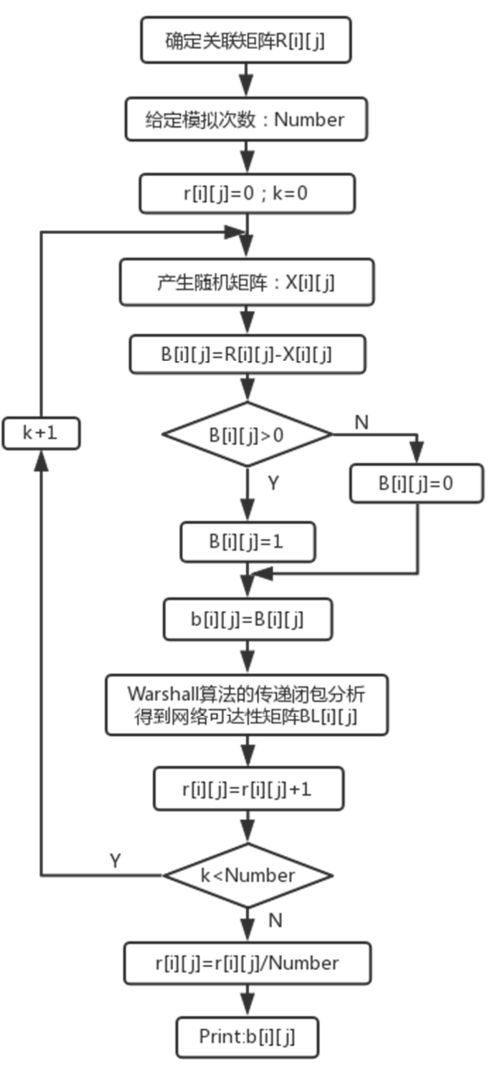

图1 蒙特卡洛法计算流程图。

\section{2. 改进蒙特卡洛法}

蒙特卡洛法的关键是求变量的随机数, 需要在 0 到 1 之间产生均匀分布的随机数, 而众所周知, 计算机产生的 随机数不是真正的自然随机数, 而是伪随机序列, 目前伪 随机数已经被证明有诸多缺陷, 如样本点存在“间隙”而减 少了它在分布上的均匀性。

因此, 改进蒙特卡洛法在传统蒙特卡洛法的基础上, 优化了随机数的生成方式, 采用准随机序列代替伪随机序 
列, 克服了由于伪随机序列抽样带来的缺点, 提高了计算 速度, 使得结果更加精确。

准随机序列在生成随机数较伪随机序列更加均匀, 而 均匀性会影响蒙特卡洛法的收敛速度以及精确度, 在文献 [12-13]中, 可知用伪随机序列的蒙特卡洛法的收玫速度为 $O\left(N^{-1 / 2}\right)$, 准随机序列的为 $\mathrm{O}\left((\log N)^{k} N^{-1}\right)$, 所以准随机 序列可以产生更加均匀的随机数。

而在准随机序列中, Sobol序列在高维的表现最好 [14], 其基本原理如下:

设 $q_{i}$ 为小于 $2^{\mathrm{i}}$ 的正奇数, 则

$$
x_{i}=\frac{q_{i}}{2^{i}}
$$

式中 $x_{i}$ 是借助如下功能多项式产生, 且只为 0 或 1 。

$$
f(z)=z^{p}+a_{1} z^{p-1}+\ldots+a_{p-1} z+a_{p}
$$

当 $i>p$ 时，有递归公式:

$$
x_{i}=a_{1} x_{i-1} \oplus a_{2} x_{i-2} \oplus \ldots \oplus a_{p} x_{i-p} \oplus \frac{x_{i-p}}{2^{p}}
$$

式子中 $\oplus$ 为二进制的异或, 按位逐个运算, 如 $8 \oplus 16=01000_{2} \oplus 10000_{2}=11000_{2}=24$ 。

对于 $q_{i}$, 当 $i>p$ 时, 同样有

$$
q_{i}=2 a_{1} x_{i-1} \oplus 2^{2} a_{2} x_{i-2} \oplus \ldots \oplus 2^{p} a_{p} x_{i-p} \oplus x_{i-p}
$$

最后便可得到Sobol序列的第 $\mathrm{n}$ 个数:

$$
s_{n}=e_{1} x_{1} \oplus e_{2} x_{2} \oplus e_{3} x_{3} \oplus \ldots
$$

式中的 $e_{1}, e_{2}, e_{3} \ldots$ 为 $\mathrm{n}$ 的二进制表示形式。

Sobol序 (点) 列的构造方法简单, 点列的均匀性较 好, 其与伪随机数序进行 100 次、 500 次抽样, 在单位平面 上的分布如图2所示[15]。
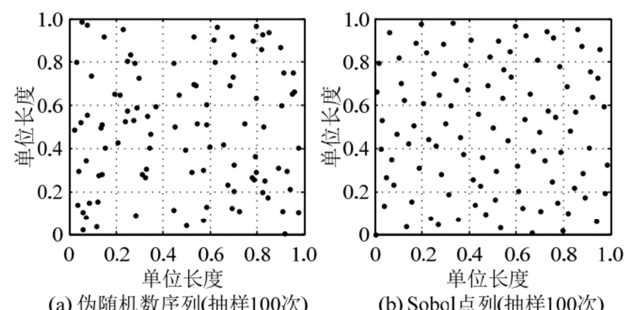

(a) 伪随机数序列(抽样 100 次)

(b) Sobol点列(抽样100次)

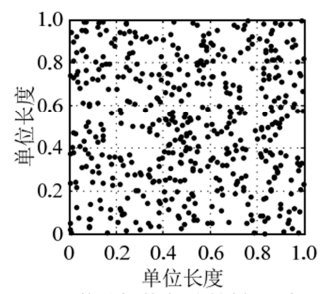

(c) 伪随机数序列(抽样500次)

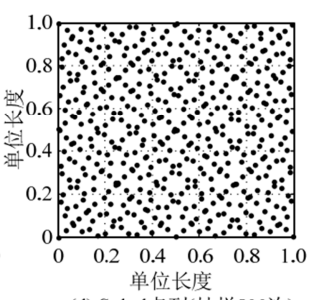

(d) Sobol点列(抽样500次)

图2 蒙特卡洛法计算流程图。
由图对比可以看出, 伪随机数序列的抽样点较Sobol 序列会有局部团簇的现象, 而Sobol则很均匀。

Sobol序列到达 $1 \%$ 精度只需要 700 次左右模拟, 而伪随 机数需要 15000 次左右。但是Sobol在 260 维内表现比较好, 超过一定维数则会发生聚集现象。而在道路网络中, 由于 其复杂和庞大, 需要采取必要的手段来增加其精度, 并且 要生成n维的矩阵非常困难。文献[16-19]从Sobol的原理出 发, 通过去掉前 1000 个点来优化 Sobol序列, 可以支持 21201 维。本文利用此工具结合连通可靠性算法, 从而解 决了道路网络高维的需求。

改进蒙特卡洛法在道路连通可靠性的计算流程如图3 所示。

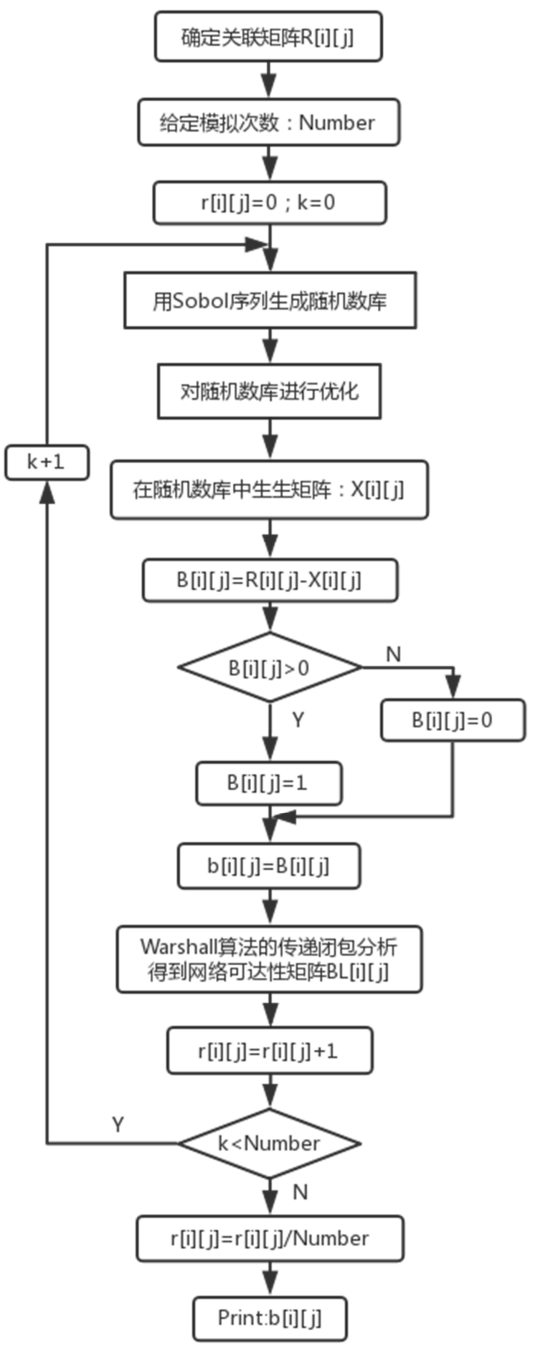

图3 改进蒙特卡洛法计算流程。

\section{3. 改进蒙特卡洛法与蒙特卡洛法比较}

对于传统的蒙特卡洛法而言, 有一些缺点: 传统的蒙 特卡罗法得样本是由伪随机数生成, 而目前伪随机数已经 被证明有诸多缺陷, 如样本点存在“间隙”而减少了它在分 布上的均匀性。并且均匀性会影响蒙特卡洛法的收敛速度 以及精确度, 所以传统的蒙特卡罗法存在收玫速度慢, 精 确度低等缺点。 
改进的蒙特卡洛法的优势在于: 改进蒙特卡洛法将低 偏差序列即其中的Sobol序列代替了伪随机数序列对系统 进行可靠性分析。与此同时, 改进蒙特卡洛法优化了Sobol 序列生成的随机数, 通过改进, 不仅提高了收玫速度和精 确度, 而且能适应复杂的道路网络, 更适合于道路的抗震 可靠性研究。

\section{3. 道路的连通可靠性计算}

\section{1. 连通可靠性算例}

以图 4 中的船型网络为例, 用蒙特卡罗法来计算网络 图中节点间的连通可靠度。

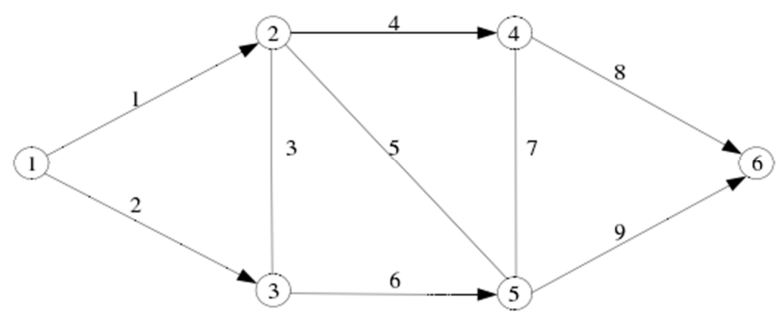

图4 船型网络图。

设所有弧其中 (弧 3 和 7 是无向弧) 的可靠度即道路连 通性为 0.7 , 节点单元的可靠度均为 1 , 则可建立关联矩阵 $\mathrm{R}[\mathrm{i}][\mathrm{j}]$ 。

$$
R[i][j]=\left[\begin{array}{cccccc}
1 & 0.7 & 0.7 & 0 & 0 & 0 \\
0 & 1 & 0.7 & 0.7 & 0.7 & 0 \\
0 & 0.7 & 1 & 0 & 0.7 & 0 \\
0 & 0 & 0 & 1 & 0.7 & 0.7 \\
0 & 0 & 0 & 0.7 & 1 & 0.7 \\
0 & 0 & 0 & 0 & 0 & 1
\end{array}\right]
$$

道路网络的可靠度可以用布尔代数中的展开定理来 分析[20],较一般的概率论分析法工作量要小很多, 而且不 需要计算机, 所以这种方法在低维时很适用。采用此法可 得到连通可靠度的精确值 $J[i][\mathrm{j}]$ 。

$$
\mathrm{J}[i][j]=\left[\begin{array}{cccccc}
1.00000 & 0.84700 & 0.84700 & 0.78285 & 0.84548 & 0.74153 \\
0 & 1.00000 & 0.70000 & 0.87787 & 0.92197 & 0.84548 \\
0 & 0.70000 & 1.00000 & 0.63357 & 0.87787 & 0.78285 \\
0 & 0 & 0 & 1.00000 & 0.70000 & 0.84700 \\
0 & 0 & 0 & 0.70000 & 1.00000 & 0.84700 \\
0 & 0 & 0 & 0 & 0 & 1.00000
\end{array}\right]
$$

\section{2. 蒙特卡洛法计算道路的连通可靠性}

Matlab作为当今国际科学计算软件的先进水平的代 表, 为众多领域的计算问题提供了全面的解决方案, 具有 强大的库, 可以用其进行道路连通可靠性的蒙特卡洛法计 算。其100次、1000次、10000次运算结果如下:

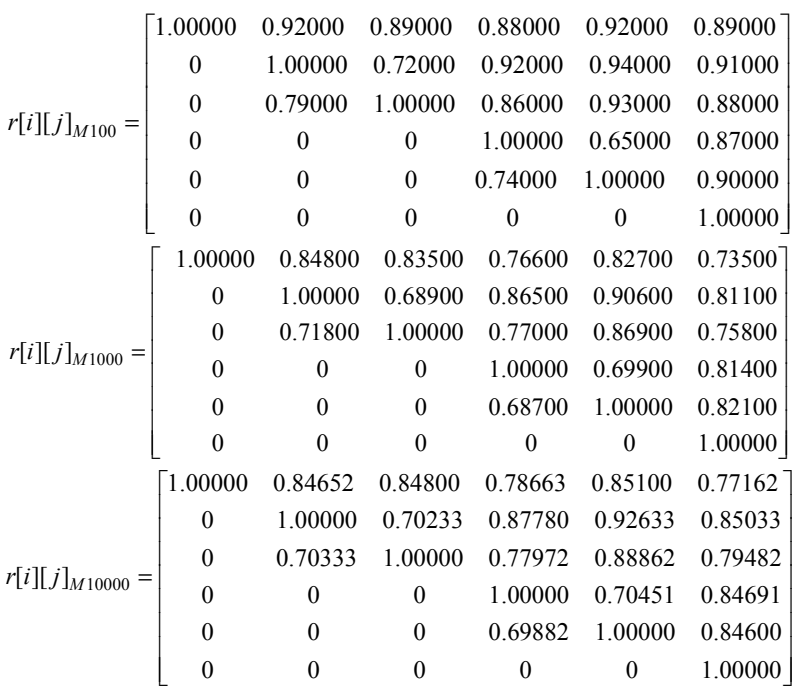

由于每次模拟的结果不一样, 可以将蒙特卡洛法的计 算结果与连通可靠度的精确值进行比较, 并计算误差。

100 次随机模拟的结果:

边 1 连通可靠度为 $92.00000 \%$, 相对误差为 $8.61865 \%$; 边 2 连通可靠度为 $89.00000 \%$, 相对误差为 $5.07674 \%$; 边 3 连通可靠度为 $72.00000 \%$, 相对误差为 $2.36128 \%$; 边 4 连通可靠度为 $92.00000 \%$, 相对误差为 $4.97403 \%$; 边 5 连通可靠度为 $94.00000 \%$, 相对误差为 $2.12869 \%$; 边6连通可靠度为 $93.00000 \%$, 相对误差为 $6.15466 \%$; 边 7 连通可靠度为 $65.00000 \%$, 相对误差为 $5.90319 \%$; 边 8 连通可靠度为 $87.00000 \%$, 相对误差为 $2.71547 \%$; 边 9 连通可靠度为 $90.00000 \%$, 相对误差为 $6.25738 \%$ 。

其相对误差的平均值为 $4.91001 \%$ ，同理， 1000 次和 10000 次的计算结果相对误差平均值为 $1.59662 \%$ 和 $0.32179 \%$ 。

可以看出, 蒙特卡洛法可以求解大型复杂网络的连通 可靠度, 而且次数愈多, 精度越高。

\section{3. 改进蒙特卡洛法计算道路的抗震可靠性}

同样以图4船型网络为例, 用Matlab进行计算, 改进 蒙特卡罗法计算网络图中节点间的连通可靠度的结果如 表1:

表1 改进蒙特卡洛法与解析法的计算误差比较。

\begin{tabular}{lllll}
\hline 模拟次数边单元 & $\mathbf{1 0 0}$ & $\mathbf{1 0 0 0}$ & $\mathbf{1 0 0 0 0}$ & 误差平均值 \\
\hline 1 & $0.70838 \%$ & $0.08560 \%$ & $0.05208 \%$ & \\
2 & $0.76741 \%$ & $0.46045 \%$ & $0.01272 \%$ & 100 次: \\
3 & $0.41322 \%$ & $0.14463 \%$ & $0.05470 \%$ & $0.70563 \%$ \\
4 & $1.43211 \%$ & $0.19244 \%$ & $0.03293 \%$ & 1000 次: \\
5 & $1.00708 \%$ & $0.23377 \%$ & $0.02427 \%$ & $0.18995 \%$ \\
6 & $0.25148 \%$ & $0.17946 \%$ & $0.01141 \%$ & 10000 次: \\
7 & $0.59032 \%$ & $0.11216 \%$ & $0.05431 \%$ & $0.02988 \%$ \\
8 & $1.00354 \%$ & $0.22727 \%$ & $0.02020 \%$ & \\
9 & $0.17710 \%$ & $0.07379 \%$ & $0.00630 \%$ & \\
\hline
\end{tabular}

从上述表格可以看出，改进蒙特卡洛法在 100 次模拟 时, 其相对误差在 $1 \%$ 左右; 当模拟次数达到 1000 次时, 其相对误差在 $0.2 \%$ 以下; 而模拟次数增加到 10000 次时, 其相对误差精度达到 $0.03 \%$ 。可以看出, 改进蒙特卡洛法 
可以较好地求解大型复杂网络的连通可靠度, 而且在精度 上是非常高的。

\section{4. 结果比较}

将蒙特卡洛法和改进蒙特卡洛法 1000 模拟的计算结 果与解析法的精确解进行比较, 结果如图5 所示。

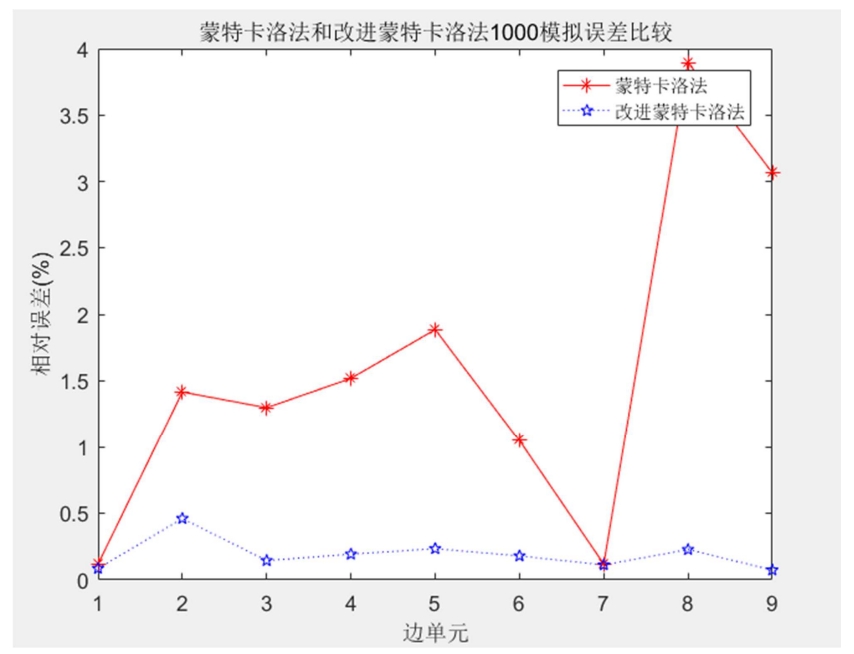

图5 蒙特卡洛法和改进蒙特卡洛法1000模拟误差比较。

从图中可以看出, 改进蒙特卡洛法的结果较传统蒙特 卡罗法数值波动较小, 且相对误差更小, 更加贴合解析法 的值, 即更加精确。一般而言, 道路网络是非常复杂且庞 大的, 所以运用改进蒙特卡罗法来分析道路的连通可靠性 更加合适。

\section{4. 结论}

本文通过改进蒙特卡洛法, 将蒙特卡洛模拟法存在的 收敛速度慢、误差具有概率性等不足的地方进行部分优化 改进, 提出一种改进的蒙特卡洛法, 即改进蒙特卡洛法。 模拟计算表明, 利用Sobol序列代替伪随机序列对路网的 连通可靠性进行计算, 更加贴近实际数值, 更加适合于精 度要求较高的大型道路网络分析。该方法在研究道路连通 可靠性上, 较传统蒙特卡洛法提高了精确度和速度, 是一 次较好的尝试。本文目前只涉及到道路可靠性的研究上, 并未涉及到其他路网连通可靠性的研究, 将在后续的工作 中做更加深入的研究。

\section{参考文献}

[1] 刘丝丝. 区域路网抗震连通可靠性研究[D].河北工业大学, 2017 。

[2] 于福莹.重大自然灾害环境下路网运行状态评估及应急保 障研究[D].燕山大学, 2016。
[3] 刘润舟.交通系统震后连通性研究[D].中国地震局工程力学 研究所, 2008。

[4] 宋永朝, 韩伟, 梁乃兴, 潘晓东, 焦建华.基于Monte Carlo 法的山区路网应急中心选址 $[\mathrm{J}]$.重庆交通大学学报(自然科 学版), 2011, 30(03):424-428。

[5] 陈玲.城市交通系统避震应急疏散线路规划方法研究[D].福 建农林大学, 2013。

[6] 潘国庆, 何明胜, 何满军.北屯市道路交通系统抗震可靠性 的分析 [J]. 石河子大学学报 (自然科学版), 2014 , 32(02):244-248。

[7] 崔姗. 区域路网抗震连通可靠性分析与优化方法[D].河北工 业大学, 2015。

[8] 付波飞.基于Monte Carlo模拟法的山地城市路网抗震应急 救护中心选址研究[D].重庆交通大学, 2016。

[9] 缪逸飞.保定市交通系统抗震可靠性分析[J]. 华南地震, 2018，38(03):62-67。

[10] 刘方荣. 不确定路网状况下的应急出救点选择问题研究 [D]. 武汉：华中科技大学，2012。

[11] 刘任任,陈建二,陈松乔.基于求传递闭包的Warshall算法的 改进[J].计算机工程,2005(19):38-39+48。

[12] Sobol I M. Quasi-Monte Carlo methods. Progress in Nuclear Energy, 1990, 24(1):55-61.

[13] Caflisch R E. Monte Carlo and quasi-Mente Carlo menthods. Acta Numerica, 1998, 7:1-49.

[14] 周心莲.几个常用随机数及其性质的比较 $[\mathrm{J}]$.勋阳师范高等 专科学校学报,2010,30(06):13-17。

[15] 侯雨伸,王秀丽,刘杰, 郭静丽,唐伦.基于拟蒙特卡罗方法的 电力系统可靠性评估[J].电网技术,2015,39(03):744-750。

[16] Bratley, P., and B. L. Fox. "Algorithm 659 Implementing Sobol's Quasirandom Sequence Generator." ACM Transactions on Mathematical Software. Vol. 14, No. 1, 1988, pp. $88-100$.

[17] Joe, S., and F. Y. Kuo. "Remark on Algorithm 659: Implementing Sobol's Quasirandom Sequence Generator." ACM Transactions on Mathematical Software. Vol. 29, No. 1, 2003, pp. 49-57.

[18] [18] Hong, H. S., and F. J. Hickernell. "Algorithm 823: Implementing Scrambled Digital Sequences." ACM Transactions on Mathematical Software. Vol. 29, No. 2, 2003, pp. 95-109.

[19] Matousek, J. "On the L2-Discrepancy for Anchored Boxes." Journal of Complexity. Vol. 14, No. 4, 1998, pp. 527-556.

[20] 何双华.供水管网系统抗震可靠性分析及加固优化研究[D]. 大连理工大学, 2009。 